УДК 517.9

\title{
A Class of Quintic Kolmogorov Systems with Explicit Non-algebraic Limit Cycle
}

\author{
Ahmed Bendjeddou \\ Department of Mathematics, Faculty of sciences \\ University of setif 1,19000 \\ Algeria \\ Mohamed Grazem* \\ Department of Mathematics, Faculty of sciences \\ University of Boumerdes, 35000
}

Algeria

Received 26.11.2018, received in revised form 29.01.2019, accepted 06.02.2019

Various physical, ecological, economic, etc phenomena are governed by planar differential systems. Subsequently, several research studies are interested in the study of limit cycles because of their interest in the understanding of these systems. The aim of this paper is to investigate a class of quintic Kolmogorov systems, namely systems of the form

$$
\begin{aligned}
& \dot{x}=x P_{4}(x, y), \\
& \dot{y}=y Q_{4}(x, y),
\end{aligned}
$$

where $P_{4}$ and $Q_{4}$ are quartic polynomials. Within this class, our attention is restricted to study the limit cycle in the realistic quadrant $\left\{(x, y) \in \mathbb{R}^{2} ; x>0, y>0\right\}$. According to the hypothesises, the existence of algebraic or non-algebraic limit cycle is proved. Furthermore, this limit cycle is explicitly given in polar coordinates. Some examples are presented in order to illustrate the applicability of our result.

Keywords: Kolmogorov systems, First integral, Periodic orbits, algebraic and non-algebraic limit cycle. DOI: 10.17516/1997-1397-2019-12-3-285-297.

\section{Introduction and preliminaries}

The so-called Kolmogorov systems on the plane are differential equations of the form

$$
\left\{\begin{array}{c}
\dot{x}=\frac{d x}{d t}=x P(x, y), \\
\dot{y}=\frac{d y}{d t}=y Q(x, y),
\end{array}\right.
$$

where $P$ and $Q$ are two coprime polynomials of $\mathbb{R}[x, y]$ and the derivatives are performed with respect to the time variable. By definition, the degree of the system (1) is the maximum of the degrees of the polynomials $P$ and $Q$. These systems arise in great variety of applications, for example, ecology and population dynamics [20,22,25], chemical reaction and plasma physics [19], hydrodynamics [10], economics, etc ...

System (1) is said to be integrable on an open set $\Omega$ of $\mathbb{R}^{2}$ if there exists a non constant continuously differentiable function $H: \Omega \longmapsto \mathbb{R}$ called a first integral of this system on $\Omega$ which

*med_grazem@univ-boumerdes.dz

(C) Siberian Federal University. All rights reserved 
is constant on the trajectories of the polynomial system (1) contained in $\Omega$ i.e., if

$$
\frac{d H}{d t}(x, y)=\frac{\partial H}{\partial x}(x, y) x P(x, y)+\frac{\partial H}{\partial y}(x, y) \text { y } Q(x, y) \equiv 0 \text { in the points of } \Omega .
$$

Moreover, $H=h$ is the general solution of the above equation, where $h$ is an arbitrary constant. It is well know that for planar differential system, the existence of a first integral determines its phase portrait, see [11].

In the qualitative theory of planar polynomial differential systems [12], one of the most important topics is related to the second part of the unsolved Hilbert 16th problem concerned essentially by the number $H(n)$ of limit cycles of (1) and their positions in the phase space. There is an extensive literature on that subject, most of it deals essentially with detection, number and stability of limit cycles.

We recall that in the phase plane, a limit cycle of system (1) is an isolated periodic solution in the set of all its periodic solutions. If limit cycle contained in the zero set of invariant algebraic curve of the plane, then we say that it is algebraic, otherwise it is called non-algebraic.

In the literature, we can find also another interesting but even more difficult problem is to give an explicit expression of a limit cycle. The limit cycles previously known in an explicit way were algebraic see $[3,4,15]$.

After the Odani's work [23], where it has been proved that the limit cycle appearing in the Van der Pol equation is not algebraic without giving an explicit expression, several articles have been published presenting differential systems polynomials for which non-algebraic limit cycles exist and are explicitly determined see $[1,6,9,14,16]$.

Concerning the Kolmogorov systems, most of the studies were limited to study the existence of limit cycles for classes of these systems see [17, 20,21,25-27]. To our knowledge, the exact analytic expressions of the limit cycles for a given Kolmogorov system is still unknown except in algebraic case. For instance, Bendjeddou, Cheurfa and Berbache in [2] showed that the quartic system admits the circle as an invariant curve which corresponds of course to the limit cycle. In the same context, Benyoucef and Bendjeddou studied in $[7,8]$ two polynomial systems of any degrees. They showed in the first paper that the considered system can admit up to four algebraic limit cycles in the plane and in the second one the system can admit a unique algebraic limit cycle in the first quadrant.

In this paper, we are interested on the quintic Kolmogorov systems of the form

where

$$
\left\{\begin{array}{l}
\dot{x}=x P_{4}(x, y), \\
\dot{y}=y Q_{4}(x, y),
\end{array}\right.
$$

$$
\begin{aligned}
P_{4}(x, y)= & 4 \lambda-4(\beta+2 \lambda) x+2(2 \beta-3 \lambda) y+2(3 \beta+4 \lambda) x^{2}+2(4 \lambda-\beta) x y+2(\lambda-2 \beta) y^{2}- \\
& -4(\beta+\lambda) x^{3}-(\beta+5 \lambda) x^{2} y+(6 \beta-2 \lambda-1) x y^{2}+(\lambda-\beta) y^{3}+ \\
& +(\beta+\lambda) x^{4}+(\beta+\lambda) x^{3} y+(1-2 \beta) x^{2} y^{2}+(\lambda-\beta) x y^{3}+(\beta-\lambda) y^{4}, \\
Q_{4}(x, y)= & -4 \lambda+2(2 \beta+\lambda) x+4(2 \lambda-\beta) y+2 \lambda x^{2}-6 \beta x y+2(3 \beta-4 \lambda) y^{2}- \\
& -3(\beta+\lambda) x^{3}+(2 \beta-2 \lambda-1) x^{2} y+(5 \beta-\lambda) x y^{2}+4(\lambda-\beta) y^{3}+ \\
& +(\beta+\lambda) x^{4}+(\beta+\lambda) x^{3} y+(1-2 \beta) x^{2} y^{2}+(\lambda-\beta) x y^{3}+(\beta-\lambda) y^{4},
\end{aligned}
$$

and $\lambda, \beta$ are reals constants. Within this class, we study the existence of a limit cycle in the realistic quadrant $\left\{(x, y) \in \mathbb{R}^{2} ; x>0, y>0\right\}$ and show under appropriate conditions that this cycle is non-algebraic giving its explicit form. 
For presenting our main result, the coordinates are translated by a vector $(1,1)$, which trasforms our system (2) to the following,

$$
\left\{\begin{array}{l}
\dot{x}=(x+1)\left(\left(x-2 y+x^{2}+x y-y^{2}\right)\left((\beta+\lambda) x^{2}+(\lambda-\beta) y^{2}\right)+x(y+1)^{2}(x+1)\right), \\
\dot{y}=(y+1)\left(\left(2 x+y+x^{2}+x y-y^{2}\right)\left((\beta+\lambda) x^{2}+(\lambda-\beta) y^{2}\right)+y(x+1)^{2}(y+1)\right) .
\end{array}\right.
$$

We can write the system (3) in polar coordinates $(r, \theta)$ through $x=r \cos \theta, y=r \sin \theta$, as

$$
\left\{\begin{array}{l}
\dot{r}=\frac{1}{8} r\left(f_{1}(\theta) r^{4}+f_{2}(\theta) r^{3}+f_{3}(\theta) r^{2}+f_{4}(\theta) r+8\right), \\
\dot{\theta}=r^{2}(\lambda+\beta \cos 2 \theta)(2+r(\cos \theta+\sin \theta))
\end{array}\right.
$$

where

$$
\begin{aligned}
& f_{1}(\theta)=1+4 \beta+4 \lambda(2 \cos 2 \theta+\sin 2 \theta)+(4 \beta-1) \cos 4 \theta+2 \beta \sin 4 \theta, \\
& f_{2}(\theta)=4((2 \beta+4 \lambda+1) \cos \theta+\sin \theta+(2 \beta-1) \cos 3 \theta+\sin 3 \theta), \\
& f_{3}(\theta)=8(1+\lambda+\beta \cos 2 \theta+2 \sin 2 \theta), \\
& f_{4}(\theta)=16(\cos \theta+\sin \theta) .
\end{aligned}
$$

Since we are dealing with solutions of system (2) in the first quadrant, we have $r \cos \theta>-1$ and $r \sin \theta>-1$ hence $(2+r(\cos \theta+\sin \theta))>0$. If $\lambda+|\beta|<\frac{-1}{2}$, then $(\lambda+\beta \cos 2 \theta)$ is strictly negative and as a result $\dot{\theta}=\frac{d \theta}{d t}$ is negative for all $t$. This signifies that $(1,1)$ is the unique equilibrium point of system (2) in the first quadrant and the orbits $(r(t), \theta(t))$ of system (4) have opposite orientation with respect to $(x(t), y(t))$ of system $(2)$.

\section{The main result}

Our main result on the limit cycles of the quintic Kolmogorov system defined by (2) is as follows

Theorem 2.1. Consider the polynomial system (2), then the following statements hold 1) If $\beta \neq 0$ and $\lambda+|\beta|<\frac{-1}{2}$, the system (2) has non-algebraic, stable and hyperbolic limit cycle explicilty given in polar coordinates $(r, \theta)$ by

$$
r\left(\theta, r_{0}\right)=\frac{A(\theta)(\cos \theta+\sin \theta)+\sqrt{A^{2}(\theta)+4 A(\theta)-A^{2}(\theta) \sin 2 \theta}}{2-A(\theta) \sin 2 \theta},
$$

where $A(\theta)=\exp (\theta)\left(\frac{r_{0}^{2}}{r_{0}+1}+\int_{0}^{\theta} \frac{\exp (-s)}{\lambda+\beta \cos 2 s} d s\right)$ and

$r_{0}=\frac{\sqrt{e^{2 \pi} \int_{0}^{2 \pi} \frac{-e^{-s}}{\lambda+\beta \cos 2 s} d s}}{2\left(e^{2 \pi}-1\right)}\left(\sqrt{e^{2 \pi} \int_{0}^{2 \pi} \frac{-e^{-s}}{\lambda+\beta \cos 2 s}} d s+\sqrt{e^{2 \pi} \int_{0}^{2 \pi} \frac{-e^{-s}}{\lambda+\beta \cos 2 s} d s+4\left(e^{2 \pi}-1\right)}\right)$.

2) If $\beta=0$ and $\lambda<\frac{-1}{2}$, the system (2) has algebraic, stable and hyperbolic limit cycle explicilty given in polar coordinates $(r, \theta)$ by $r\left(\theta, r_{0}\right)=\frac{(\cos \theta+\sin \theta)+\sqrt{1-4 \lambda-\sin 2 \theta}}{-(2 \lambda+\sin 2 \theta)}$, and in Cartesian coordinates by $\lambda(x-1)^{2}+\lambda(y-1)^{2}+x y=0$. 
For the demonstration of Theorem (2.1), we need the following lemmas

Lemma 2.2. The system of the form

$$
\left\{\begin{aligned}
\dot{r} & =F(\theta) H(r, \theta)-\frac{\partial H}{\partial \theta}(r, \theta)+G(\theta), \\
\dot{\theta} & =\frac{\partial H}{\partial r}(r, \theta)
\end{aligned}\right.
$$

possess a first integral expressed as

$$
L(r, \theta)=H(r, \theta) \exp \left(-\int_{0}^{\theta} F(s) d s\right)-\int_{0}^{\theta} G(s) \exp \left(-\int_{0}^{s} F(w) d w\right) d s .
$$

Proof. Let set $A(r, \theta)=H(r, \theta) \exp \left(-\int_{0}^{\theta} F(s) d s\right)$ and $B(\theta)=\int_{0}^{\theta} G(s) \exp \left(-\int_{0}^{s} F(w) d w\right) d s$, then the derivatives of $A$ and $B$ with respect to $\theta$ are

$$
\begin{aligned}
& \frac{d A}{d \theta}(r, \theta)=\left(\frac{\partial H}{\partial \theta}(r, \theta)-F(\theta) H(r, \theta)\right) \exp \left(-\int_{0}^{\theta} F(s) d s\right) \\
& \frac{d B}{d \theta}(\theta)=G(\theta) \exp \left(-\int_{0}^{\theta} F(s) d s\right)
\end{aligned}
$$

By replacing the expression of derivatives of $A$ and $B$ with respect to $\theta$ in the expression of $L$, it follows that $\frac{d L}{d \theta}(r, \theta)=\left(\frac{\partial H}{\partial \theta}(r, \theta)-F(\theta) H(r, \theta)-G(\theta)\right) \exp \left(-\int_{0}^{\theta} F(s) d s\right)$.

By the chain rule, the derivative of $L$ with respect to $t$ is given by following expression,

$$
\begin{aligned}
\frac{d L}{d t}(r(t), \theta(t)) & =\frac{\partial L}{\partial r}(r, \theta) \frac{d r}{d t}+\frac{\partial L}{\partial \theta}(r, \theta) \frac{d \theta}{d t}= \\
& =\left(\frac{\partial H}{\partial r}(r, \theta) \exp \left(-\int_{0}^{\theta} F(s) d s\right)\right)\left(F(\theta) H(r, \theta)-\frac{\partial H}{\partial \theta}(r, \theta)+G(\theta)\right)+ \\
& +\left(\left(\frac{\partial H}{\partial \theta}(r, \theta)-F(\theta) H(r, \theta)-G(\theta)\right) \exp \left(-\int_{0}^{\theta} F(s) d s\right)\right) \frac{\partial H}{\partial r}(r, \theta)=0
\end{aligned}
$$

So $L(r, \theta)$ is a first integral of system

Lemma 2.3. Let $\lambda, \beta \in \mathbb{R}$ such that $\lambda+|\beta|<\frac{-1}{2}$, then the following statements hold

1) $0<\frac{e^{2 \pi}}{e^{2 \pi}-1} \int_{0}^{2 \pi} \frac{-e^{-s}}{\lambda+\beta \cos 2 s} d s<2$.

2) The function $g$ defined on $[0,2 \pi]$ by $g(\theta)=2 \exp (-\theta)+\int_{0}^{\theta} \frac{-e^{-s}}{\lambda+\beta \cos 2 s} d s$ is strictly decreasing.

Furthermore $g(\theta)>\frac{e^{2 \pi}}{e^{2 \pi}-1} \int_{0}^{2 \pi} \frac{-e^{-s}}{\lambda+\beta \cos 2 s} d s$.

3) $0<A(\theta)=\exp (\theta)\left(\frac{e^{2 \pi}}{e^{2 \pi}-1} \int_{0}^{2 \pi} \frac{-e^{-s}}{\lambda+\beta \cos 2 s} d s+\int_{0}^{\theta} \frac{e^{-s}}{\lambda+\beta \cos 2 s} d s\right)<2$.

Proof of statement 1) of Lemma 2.3 We have $\lambda+\beta \cos 2 s \leqslant \lambda+|\beta|<\frac{-1}{2}$ which implies $0<$ $\frac{-e^{-s}}{\lambda+\beta \cos 2 s}<2 e^{-s}$, consequently $0<\frac{e^{2 \pi}}{e^{2 \pi}-1} \int_{0}^{2 \pi} \frac{-e^{-s}}{\lambda+\beta \cos 2 s} d s<\frac{2 e^{2 \pi}}{e^{2 \pi}-1} \int_{0}^{2 \pi} e^{-s} d s$, whence

$$
0<\frac{e^{2 \pi}}{e^{2 \pi}-1} \int_{0}^{2 \pi} \frac{-e^{-s}}{\lambda+\beta \cos 2 s} d s<2
$$


Proof of statement 2) of Lemma 2.3. Over the interval $[0,2 \pi]$, the function $\mathrm{g}$ is differentiable and

$$
g^{\prime}(\theta)=-2 \exp (-\theta)+\frac{-\exp (-\theta)}{\lambda+\beta \cos 2 \theta}=-\exp (-\theta)\left(2+\frac{1}{\lambda+\beta \cos 2 \theta}\right) .
$$

Since $\lambda+\beta \cos 2 \theta \leqslant \lambda+|\beta|$, then $g^{\prime}(\theta) \leqslant-\exp (-\theta)\left(2+\frac{1}{\lambda+|\beta|}\right)<0$. Therefore $g$ is strictly decreasing function. On the other hand, from the statement 1) of Lemma 2.3, we have $\frac{1}{e^{2 \pi}-1} \int_{0}^{2 \pi} \frac{-e^{-s}}{\lambda+\beta \cos 2 s} d s<2 e^{-2 \pi}$, which implies

$$
\frac{e^{2 \pi}}{e^{2 \pi}-1} \int_{0}^{2 \pi} \frac{-e^{-s}}{\lambda+\beta \cos 2 s} d s-\int_{0}^{2 \pi} \frac{-e^{-s}}{\lambda+\beta \cos 2 s} d s<2 e^{-2 \pi}
$$

because $\frac{1}{e^{2 \pi}-1}=\left(\frac{e^{2 \pi}}{e^{2 \pi}-1}-1\right)$, consequently

$$
\frac{e^{2 \pi}}{e^{2 \pi}-1} \int_{0}^{2 \pi} \frac{-e^{-s}}{\lambda+\beta \cos 2 s} d s<2 e^{-2 \pi}+\int_{0}^{2 \pi} \frac{-e^{-s}}{\lambda+\beta \cos 2 s} d s=g(2 \pi)
$$

as $g$ is strictly decreasing function, then $\frac{e^{2 \pi}}{e^{2 \pi}-1} \int_{0}^{2 \pi} \frac{-e^{-s}}{\lambda+\beta \cos 2 s} d s<g(\theta)$.

Proof of statement 3) of Lemma 2.3. Let us first show that $A$ is strictly positive. From the relationship of Chasles

$$
A(\theta)=\exp (\theta)\left(\frac{e^{2 \pi}}{e^{2 \pi}-1}\left(\int_{0}^{\theta} \frac{-e^{-s}}{\lambda+\beta \cos 2 s} d s+\int_{\theta}^{2 \pi} \frac{-e^{-s}}{\lambda+\beta \cos 2 s} d s\right)-\int_{0}^{\theta} \frac{-e^{-s}}{\lambda+\beta \cos 2 s} d s\right),
$$

which implies

$$
A(\theta)=\exp (\theta)\left(\frac{e^{2 \pi}}{e^{2 \pi}-1} \int_{\theta}^{2 \pi} \frac{-e^{-s}}{\lambda+\beta \cos 2 s} d s+\left(\frac{e^{2 \pi}}{e^{2 \pi}-1}-1\right) \int_{0}^{\theta} \frac{-e^{-s}}{\lambda+\beta \cos 2 s} d s\right) .
$$

Since $\frac{e^{2 \pi}}{e^{2 \pi}-1}-1>0$ and $\frac{-e^{-s}}{\lambda+\beta \cos 2 s}>0$, then $A(\theta)>0$.

Let us now show that $A(\theta)<2$. From the statement 2) of Lemma 2.3, we have

$$
\frac{e^{2 \pi}}{e^{2 \pi}-1} \int_{0}^{2 \pi} \frac{-e^{-s}}{\lambda+\beta \cos 2 s} d s<2 \exp (-\theta)+\int_{0}^{\theta} \frac{-e^{-s}}{\lambda+\beta \cos 2 s} d s,
$$

which implies $\left(\frac{e^{2 \pi}}{e^{2 \pi}-1} \int_{0}^{2 \pi} \frac{-e^{-s}}{\lambda+\beta \cos 2 s} d s+\int_{0}^{\theta} \frac{e^{-s}}{\lambda+\beta \cos 2 s} d s\right)<2 \exp (-\theta)$, therefore

$$
A(\theta)=\exp (\theta)\left(\frac{e^{2 \pi}}{e^{2 \pi}-1} \int_{0}^{2 \pi} \frac{-e^{-s}}{\lambda+\beta \cos 2 s} d s+\int_{0}^{\theta} \frac{e^{-s}}{\lambda+\beta \cos 2 s} d s\right)<2,
$$

whence $0<A(\theta)<2$.

Proof of Theorem 2.1. We assume that $\lambda+|\beta|<\frac{-1}{2}$. In the new independent variable $\theta$, the differential system (4) becomes

$$
\frac{d r}{d \theta}=\frac{1}{8 r} \frac{f_{1}(\theta) r^{4}+f_{2}(\theta) r^{3}+f_{3}(\theta) r^{2}+f_{4}(\theta) r+8}{(\lambda+\beta \cos 2 \theta)(2+r(\cos \theta+\sin \theta))},
$$


which can be expressed as

$$
\frac{d r}{d \theta}=\frac{F(\theta) H(r, \theta)-\frac{\partial H}{\partial \theta}(r, \theta)+G(\theta)}{\frac{\partial H}{\partial r}(r, \theta)}
$$

where $H(r, \theta)=\frac{r^{2}}{(r \cos \theta+1)(r \sin \theta+1)}, \quad F(\theta)=1$ and $G(\theta)=\frac{1}{\lambda+\beta \cos 2 \theta}$.

By Lemma 2.2, the solutions of the equation (7) are expressed as

$$
\frac{r^{2}}{(r \cos \theta+1)(r \sin \theta+1)}-\exp (\theta)\left(k+\int_{0}^{\theta} \frac{e^{-s}}{\lambda+\beta \cos 2 s} d s\right)=0, \quad \text { where } k \in \mathbb{R} .
$$

In the region $2-A(\theta) \sin 2 \theta \neq 0$ and $A^{2}(\theta)+4 A(\theta)-A^{2}(\theta) \sin 2 \theta \geqslant 0$ the equation (9) has two solutions

$$
r_{1,2}(\theta)=\frac{A(\theta)(\cos \theta+\sin \theta) \pm \sqrt{A^{2}(\theta)+4 A(\theta)-A^{2}(\theta) \sin 2 \theta}}{2-A(\theta) \sin 2 \theta},
$$

with $A(\theta)=\exp (\theta)\left(k+\int_{0}^{\theta} \frac{e^{-s}}{\lambda+\beta \cos 2 s} d s\right)$.

Notice that system (3) has a periodic solution if and only if equation (7) has a strictly positive $2 \pi$-periodic solution. For $\theta=0$, we have

$$
r_{1}(0)=\frac{1}{2}(k+\sqrt{k(k+4)}) \text { and } r_{2}(0)=\frac{1}{2}(k-\sqrt{k(k+4)}),
$$

$r_{1,2}(0)$ are defined if only if $\left.k \in\right]-\infty,-4[\cup] 0,+\infty[$. Over the interval $]-\infty,-4\left[, r_{1}(0)\right.$ and $r_{2}(0)$ are negative, and in $] 0,+\infty\left[, r_{1}(0)\right.$ is positive but $r_{2}(0)$ is negative. Consequently, the admitted solution of equation (9) is

$$
r(\theta)=r_{1}(\theta)=\frac{A(\theta)(\cos \theta+\sin \theta)+\sqrt{A^{2}(\theta)+4 A(\theta)-A^{2}(\theta) \sin 2 \theta}}{2-A(\theta) \sin 2 \theta} .
$$

where $A(\theta)=\exp (\theta)\left(k+\int_{0}^{\theta} \frac{e^{-s}}{\lambda+\beta \cos 2 s} d s\right)$ and $k=\frac{r^{2}(0)}{r(0)+1}>0$.

The solution of the equation (9) starting at $r\left(0, r_{0}\right)=r_{0}>0$ is given by

$$
r\left(\theta, r_{0}\right)=\frac{A(\theta)(\cos \theta+\sin \theta)+\sqrt{A^{2}(\theta)+4 A(\theta)-A^{2}(\theta) \sin 2 \theta}}{2-A(\theta) \sin 2 \theta},
$$

where $A(\theta)=\exp (\theta)\left(\frac{r_{0}^{2}}{r_{0}+1}+\int_{0}^{\theta} \frac{e^{-s}}{\lambda+\beta \cos 2 s} d s\right)$ and $r_{0}=r(0)$.

The condition of the periodic solution with $2 \pi$-periodic starting at $r\left(0, r_{0}\right)=r_{0}>0$ is $r\left(0, r_{0}\right)=r\left(2 \pi, r_{0}\right)$. For $\theta=2 \pi$, we obtain

$$
r\left(2 \pi, r_{0}\right)=\frac{1}{2}(A(2 \pi)+\sqrt{A(2 \pi)(A(2 \pi)+4)})
$$

where $A(2 \pi)=e^{2 \pi}\left(\frac{r_{0}^{2}}{r_{0}+1}+\int_{0}^{2 \pi} \frac{e^{-s}}{\lambda+\beta \cos 2 s} d s\right)$. 
The resolution of equation $r\left(0, r_{0}\right)=r\left(2 \pi, r_{0}\right)$ gives

$$
r_{0}=\frac{\sqrt{e^{2 \pi} \int_{0}^{2 \pi} \frac{-e^{-s}}{\lambda+\beta \cos 2 s}} d s}{2\left(e^{2 \pi}-1\right)}\left(\sqrt{e^{2 \pi} \int_{0}^{2 \pi} \frac{-e^{-s}}{\lambda+\beta \cos 2 s}} d s+\sqrt{e^{2 \pi} \int_{0}^{2 \pi} \frac{-e^{-s}}{\lambda+\beta \cos 2 s} d s+4\left(e^{2 \pi}-1\right)}\right) .
$$

By some simplifications, we obtain $\frac{r_{0}^{2}}{r_{0}+1}=\frac{e^{2 \pi}}{e^{2 \pi}-1} \int_{0}^{2 \pi} \frac{-e^{-s}}{\lambda+\beta \cos 2 s} d s$. Finally, the general solution of (4) is given explicitly by

$$
r\left(\theta, r_{0}\right)=\frac{A(\theta)(\cos \theta+\sin \theta)+\sqrt{A^{2}(\theta)+4 A(\theta)-A^{2}(\theta) \sin 2 \theta}}{2-A(\theta) \sin 2 \theta},
$$

with $A(\theta)=\exp (\theta)\left(\frac{e^{2 \pi}}{e^{2 \pi}-1} \int_{0}^{2 \pi} \frac{-e^{-s}}{\lambda+\beta \cos 2 s} d s+\int_{0}^{\theta} \frac{e^{-s}}{\lambda+\beta \cos 2 s} d s\right)$ and $r_{0}=r(0)$.

Periodicity of $r\left(\theta, r_{0}\right)$. Let us now show that $A(\theta)$ is $2 \pi$-periodic function. We have

$$
A(\theta+2 \pi)=\exp (\theta+2 \pi)\left(\frac{e^{2 \pi}}{e^{2 \pi}-1} \int_{0}^{2 \pi} \frac{-e^{-s}}{\lambda+\beta \cos 2 s} d s+\int_{0}^{\theta+2 \pi} \frac{e^{-s}}{\lambda+\beta \cos 2 s} d s\right),
$$

it follows

$A(\theta+2 \pi)=e^{\theta+2 \pi}\left(\frac{e^{2 \pi}}{e^{2 \pi}-1} \int_{0}^{2 \pi} \frac{-e^{-s}}{\lambda+\beta \cos 2 s} d s+\int_{0}^{2 \pi} \frac{e^{-s}}{\lambda+\beta \cos 2 s} d s+\int_{2 \pi}^{\theta+2 \pi} \frac{e^{-s}}{\lambda+\beta \cos 2 s} d s\right)$ i.e.

$$
A(\theta+2 \pi)=e^{\theta} e^{2 \pi}\left(\frac{1}{e^{2 \pi}-1} \int_{0}^{2 \pi} \frac{-e^{-s}}{\lambda+\beta \cos 2 s} d s+\int_{2 \pi}^{\theta+2 \pi} \frac{e^{-s}}{\lambda+\beta \cos 2 s} d s\right)
$$

by the change of variable $u=s-2 \pi$, we obtain $\int_{2 \pi}^{\theta+2 \pi} \frac{e^{-s}}{\lambda+\beta \cos 2 s} d s=e^{-2 \pi} \int_{0}^{\theta} \frac{e^{-s}}{\lambda+\beta \cos 2 s} d s$, then

$$
A(\theta+2 \pi)=e^{\theta}\left(\frac{e^{2 \pi}}{e^{2 \pi}-1} \int_{0}^{2 \pi} \frac{-e^{-s}}{\lambda+\beta \cos 2 s} d s+e^{2 \pi} e^{-2 \pi} \int_{0}^{\theta} \frac{e^{-s}}{\lambda+\beta \cos 2 s} d s\right),
$$

therefore $A(\theta+2 \pi)=A(\theta)$. Furthermore, as $\theta \longmapsto \sin \theta, \theta \longmapsto \cos \theta$ and $\theta \longmapsto A(\theta)$ are $2 \pi$-periodic functions, then $r\left(\theta, r_{0}\right)$ is also.

Strict positivity of $r\left(\theta, r_{0}\right)$. By the statement 3) of Lemma 2.3, we have $0<A(\theta)<2$, then the denominator of $r\left(\theta, r_{0}\right)$ is strictly positive. Two cases are distinguished

i) If $(\cos \theta+\sin \theta)>0$, the numerator of $r\left(\theta, r_{0}\right)$ is strictly positive, consequently $r\left(\theta, r_{0}\right)$ is also.

ii) If $(\cos \theta+\sin \theta)<0$, we have $4 A(\theta)-2 A^{2}(\theta) \sin 2 \theta>0$, which implies

$$
A^{2}(\theta)+4 A(\theta)-A^{2}(\theta) \sin 2 \theta>A(\theta)+A^{2}(\theta) \sin 2 \theta,
$$

i.e.

$$
A^{2}(\theta)+4 A(\theta)-A^{2}(\theta) \sin 2 \theta>(-A(\theta)(\cos \theta+\sin \theta))^{2}
$$

then

$$
\sqrt{A^{2}(\theta)+4 A(\theta)-A^{2}(\theta) \sin 2 \theta}>-A(\theta)(\cos \theta+\sin \theta),
$$


hence

$$
A(\theta)(\cos \theta+\sin \theta)+\sqrt{A^{2}(\theta)+4 A(\theta)-A^{2}(\theta) \sin 2 \theta}>0 .
$$

Therefore $r\left(\theta, r_{0}\right)$ is stictly positive. Finally $r\left(\theta, r_{0}\right)$ defines through (4) is a periodic solution. Let us show that this peiodic solution is a limit cycle. For this aim, we introduce the Poincaré return map

$$
\gamma \mapsto \Pi(\gamma)=r(2 \pi, \gamma)=\frac{1}{2}\left(A(2 \pi)+\sqrt{A^{2}(2 \pi)+4 A(2 \pi)}\right),
$$

where $A(2 \pi)=\exp (2 \pi)\left(\frac{\gamma^{2}}{\gamma+1}+\int_{0}^{2 \pi} \frac{e^{-s}}{\lambda+\beta \cos 2 s} d s\right)$ and show that the function of Poincare first return verify $\left.\frac{d \Pi(\gamma)}{d \gamma}\right|_{\gamma=r_{0}} \neq 1$ see [12]. We remark that

$$
\sqrt{A^{2}(2 \pi)+4 A(2 \pi)}=\frac{e^{\pi}}{(\gamma+1)} \sqrt{\left(\gamma^{2}+D \gamma+D\right)\left(e^{2 \pi}\left(\gamma^{2}+D \gamma+D\right)+4(\gamma+1)\right)}
$$

with

We have

$$
D=\int_{0}^{2 \pi} \frac{e^{-s}}{\lambda+\beta \cos 2 s} d s
$$

$$
\frac{\partial}{\partial \gamma}(A(2 \pi))=\frac{\gamma(\gamma+2)}{(\gamma+1)^{2}} e^{2 \pi}
$$

and

$$
\begin{aligned}
& \frac{\partial}{\partial \gamma}\left(\frac{e^{\pi}}{(\gamma+1)} \sqrt{\left(\gamma^{2}+D \gamma+D\right)\left(e^{2 \pi}\left(\gamma^{2}+D \gamma+D\right)+4(\gamma+1)\right)}\right)= \\
& =\frac{\gamma(\gamma+2) e^{\pi}}{(\gamma+1)^{2}} \frac{\left(e^{2 \pi}\left(\gamma^{2}+D \gamma+D\right)+2(\gamma+1)\right)}{\sqrt{\left(\gamma^{2}+D \gamma+D\right)\left(e^{2 \pi}\left(\gamma^{2}+D \gamma+D\right)+4(\gamma+1)\right)}}
\end{aligned}
$$

consequently

$$
\begin{aligned}
& \frac{\partial}{\partial \gamma}\left(\frac{1}{2}\left(A(2 \pi)+\sqrt{A^{2}(2 \pi)+4 A(2 \pi)}\right)\right)= \\
& =\frac{1}{2} \frac{\gamma(\gamma+2) e^{\pi}}{(\gamma+1)^{2}}\left(\frac{\left(e^{2 \pi}\left(\gamma^{2}+D \gamma+D\right)+2(\gamma+1)\right)}{\sqrt{\left(\gamma^{2}+D \gamma+D\right)\left(e^{2 \pi}\left(\gamma^{2}+D \gamma+D\right)+4(\gamma+1)\right)}}+e^{\pi}\right)
\end{aligned}
$$

then

$$
\begin{aligned}
\left.\frac{d \Pi(\gamma)}{\gamma}\right|_{\gamma=r_{0}} & =\left.\frac{1}{2} \frac{\gamma(\gamma+2) e^{\pi}}{(\gamma+1)^{2}}\left(\frac{\left(e^{2 \pi}\left(\gamma^{2}+D \gamma+D\right)+2(\gamma+1)\right)}{\sqrt{\left(\gamma^{2}+D \gamma+D\right)\left(e^{2 \pi}\left(\gamma^{2}+D \gamma+D\right)+4(\gamma+1)\right)}}+e^{\pi}\right)\right|_{\gamma=r_{0}}= \\
& =\frac{1}{2} \frac{r_{0}\left(r_{0}+2\right) e^{\pi}}{\left(r_{0}+1\right)^{2}}\left(\frac{\left(e^{2 \pi}\left(r_{0}^{2}+D r_{0}+D\right)+2\left(r_{0}+1\right)\right)}{\sqrt{\left(r_{0}^{2}+D r_{0}+D\right)\left(e^{2 \pi}\left(r_{0}^{2}+D r_{0}+D\right)+4\left(r_{0}+1\right)\right)}}+e^{\pi}\right)
\end{aligned}
$$

Since $\lambda+|\beta|<\frac{-1}{2}$, then $0<\left(r_{0}^{2}+D r_{0}+D\right)<r_{0}^{2}$ because $D<0$ and $A>0$. Therefore

$$
\left(e^{2 \pi}\left(r_{0}^{2}+D r_{0}+D\right)+4\left(r_{0}+1\right)\right)<\left(e^{2 \pi} r_{0}^{2}+4\left(r_{0}+1\right)\right)<e^{2 \pi}\left(r_{0}^{2}+2 r_{0}+1\right)=e^{2 \pi}\left(r_{0}+1\right)^{2}
$$

because $e^{2 \pi}>4$. It follows that

$$
\sqrt{\left(r_{0}^{2}+D r_{0}+D\right)\left(e^{2 \pi}\left(r_{0}^{2}+D r_{0}+D\right)+4\left(r_{0}+1\right)\right)}<\sqrt{r_{0}^{2} e^{2 \pi}\left(r_{0}+1\right)^{2}}=e^{\pi} r_{0}\left(r_{0}+1\right),
$$


i.e. $\frac{1}{\sqrt{\left(r_{0}^{2}+D r_{0}+D\right)\left(e^{2 \pi}\left(r_{0}^{2}+D r_{0}+D\right)+4\left(r_{0}+1\right)\right)}}>\frac{1}{e^{\pi} r_{0}\left(r_{0}+1\right)}$, which implies

$$
\frac{\left(e^{2 \pi}\left(r_{0}^{2}+D r_{0}+D\right)+2\left(r_{0}+1\right)\right)}{\sqrt{\left(r_{0}^{2}+D r_{0}+D\right)\left(e^{2 \pi}\left(r_{0}^{2}+D r_{0}+D\right)+4\left(r_{0}+1\right)\right)}}>\frac{2\left(r_{0}+1\right)}{e^{\pi} r_{0}\left(r_{0}+1\right)}=\frac{2}{e^{\pi} r_{0}},
$$

because $\left(e^{2 \pi}\left(r_{0}^{2}+D r_{0}+D\right)+2\left(r_{0}+1\right)\right)>2\left(r_{0}+1\right)$.

Consequently

$$
\begin{aligned}
\left.\frac{d \Pi(\gamma)}{d \gamma}\right|_{\gamma=r_{0}} & >\frac{1}{2} \frac{r_{0}\left(r_{0}+2\right) e^{\pi}}{\left(r_{0}+1\right)^{2}}\left(\frac{2}{e^{\pi} r_{0}}+e^{\pi}\right)= \\
& =\frac{\left(r_{0}+2\right)}{\left(r_{0}+1\right)^{2}}+\frac{1}{2} \frac{r_{0}\left(r_{0}+2\right)}{\left(r_{0}+1\right)^{2}} e^{2 \pi}= \\
& =\frac{\left(r_{0}+2\right)}{\left(r_{0}+1\right)^{2}}\left(1+\frac{e^{2 \pi}}{2} r_{0}\right)>\left(\text { because } \frac{e^{2 \pi}}{2}>1\right) \\
& >\frac{\left(r_{0}+2\right)}{\left(r_{0}+1\right)^{2}}\left(1+r_{0}\right)= \\
& =\frac{\left(r_{0}+2\right)}{\left(r_{0}+1\right)}>1 .
\end{aligned}
$$

Hence

$$
\left.\frac{d \Pi(\gamma)}{d \gamma}\right|_{\gamma=r_{0}}>1
$$

Therefore the solution of differential equation (4) is unstable and hyperbolic limit cycle see [12], consequently, it is a stable and hyperbolic limit cycle for the system (2).

1) If $\beta \neq 0$, this limit cycle is non-algebraic, due to the expression of $A(\theta)$.

More precisely, in Cartesian coordinates $\left(r^{2}=(x-1)^{2}+(y-1)^{2}, \theta=\arctan \left(\frac{y-1}{x-1}\right)\right)$, the curve defined by this limit cycle is $f(x, y)=\frac{(x-1)^{2}+(y-1)^{2}}{x y}-B(x, y)=0$, with

$$
B(x, y)=\exp \left(\arctan \left(\frac{y-1}{x-1}\right)\right)\left(\frac{e^{2 \pi}}{e^{2 \pi}-1}\left(\int_{0}^{2 \pi} \frac{-e^{-s}}{\lambda+\beta \cos 2 s} d s\right)+\int_{0}^{\arctan \frac{y-1}{x-1}} \frac{e^{-s}}{\lambda+\beta \cos 2 s} d s\right) .
$$

There is no integer $n$ for which both $\frac{\partial^{n} f}{\partial x^{n}}$ and $\frac{\partial^{n} f}{\partial y^{n}}$ vanish identically. To be convinced by this fact, one has compute for example $\frac{\partial f}{\partial y}$, that is

$$
\frac{\partial f}{\partial y}(x, y)=\frac{-x^{2}+2 x+y^{2}-2}{x y^{2}}-\frac{x-1}{(x-1)^{2}+(y-1)^{2}}\left[B(x, y)+\frac{1}{\lambda+\beta \cos \left(2 \arctan \left(\frac{y-1}{x-1}\right)\right)}\right] .
$$

Since $B(x, y)$ appears again, it will remains in any order of derivation, therefore the curve $f(x, y)=0$ is non-algebraic and the limit cycle of the system (2) will also be non-algebraic. This complete the proof of statement 1) of Theorem 2.1.

2) If $\beta=0$, we have $\int_{0}^{\theta} \frac{\exp (-s)}{\lambda} d s=\frac{1}{\lambda}\left(1-e^{-\theta}\right)$ and $e^{2 \pi} \int_{0}^{2 \pi} \frac{-e^{-s}}{\lambda} d s=\frac{1}{\lambda}\left(1-e^{2 \pi}\right)$, by simplification we obtain $r_{0}=\frac{-1}{2 \lambda}(\sqrt{1-4 \lambda}+1)$ and $\frac{r_{0}^{2}}{r_{0}+1}=\frac{-1}{\lambda}=A(\theta)$. By substituting the values of $r_{0}$ and $A(\theta)$ in (12), the solution of (4) becomes 


$$
r\left(\theta, r_{0}\right)=\frac{\frac{-1}{\lambda}(\cos \theta+\sin \theta)+\sqrt{\frac{1}{\lambda^{2}}-\frac{4}{\lambda}-\frac{1}{\lambda^{2}} \sin 2 \theta}}{2+\frac{1}{\lambda} \sin 2 \theta}=\frac{(\cos \theta+\sin \theta)+\sqrt{1-4 \lambda-\sin 2 \theta}}{-(2 \lambda+\sin 2 \theta)} .
$$

In Cartesian coordinates, the curve defined by this limit cycle is $\lambda(x-1)^{2}+\lambda(y-1)^{2}+x y=0$ which is algebraic. This complete the proof of statement 2) of Theorem 2.1.

\section{Applications}

In this section, we present some examples to illustrate the applicability of the our main result. In addition, a plot of phase portraits in the Poincaré disc for each example were performed showing a limit cycle in the first quadrant.

Eexample 3.1. In the system (2), we take $\lambda=-2$ and $\beta=1\left(\lambda+|\beta|=-1<\frac{-1}{2}\right)$, we obtain

$$
\left\{\begin{array}{c}
\dot{x}=x\left(\begin{array}{l}
-8+12 x+16 y-10 x^{2}-18 x y-8 y^{2}+4 x^{3}+9 x^{2} y \\
+9 x y^{2}-3 y^{3}-x^{4}-x^{3} y-x^{2} y^{2}-3 x y^{3}+3 y^{4}
\end{array}\right), \\
\dot{y}=y\left(\begin{array}{l}
8-20 y-4 x^{2}-6 x y+22 y^{2}+3 x^{3}+5 x^{2} y \\
+7 x y^{2}-12 y^{3}-x^{4}-x^{3} y-x^{2} y^{2}-3 x y^{3}+3 y^{4}
\end{array}\right)
\end{array}\right.
$$

which has a non-algebraic, stable and hyperbolic limit cycle whose expression in polar coordinates $(r, \theta)$ is

$$
r\left(\theta, r_{0}\right)=\frac{A(\theta)(\cos \theta+\sin \theta)+\sqrt{A^{2}(\theta)+4 A(\theta)-A^{2}(\theta) \sin 2 \theta}}{2-A(\theta) \sin 2 \theta},
$$

where $A(\theta)=\exp (\theta)\left(\frac{r_{0}^{2}}{r_{0}+1}+\int_{0}^{\theta} \frac{\exp (-s)}{-2+\cos 2 s} d s\right)$ and $r_{0} \simeq 1.1877$ (Fig. 1).

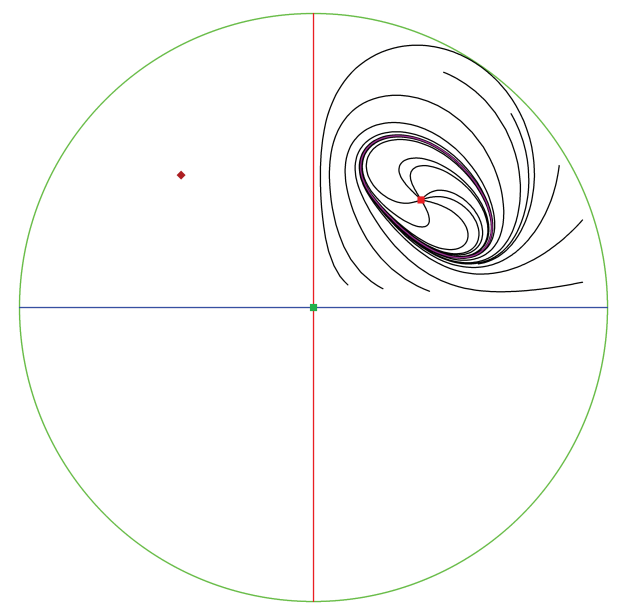

Fig. 1. The phase portrait on the Poincaré disc of the system (13), showing a limit cycles in the first quadrant 
Eexample 3.2. In the system (2), we take $\lambda=-10$ and $\beta=0\left(\lambda<\frac{-1}{2}\right)$, we obtain

$$
\left\{\begin{array}{c}
\dot{x}=x\left(\begin{array}{l}
-40+80 x+60 y-80 x^{2}-80 x y-20 y^{2}+40 x^{3}+50 x^{2} y \\
+19 x y^{2}-10 y^{3}-10 x^{4}-10 x^{3} y+x^{2} y^{2}-10 x y^{3}+10 y^{4}
\end{array}\right), \\
\dot{y}=y\left(\begin{array}{l}
40-20 x-80 y-20 x^{2}+80 y^{2}+30 x^{3}+19 x^{2} y+10 x y^{2} \\
-40 y^{3}-10 x^{4}-10 x^{3} y+x^{2} y^{2}-10 x y^{3}+10 y^{4}
\end{array}\right),
\end{array}\right.
$$

which has an algebraic, stable and hyperbolic limit cycle given by the expression (Fig. 2).

$$
-10(x-1)^{2}-10(y-1)^{2}+x y=0 .
$$

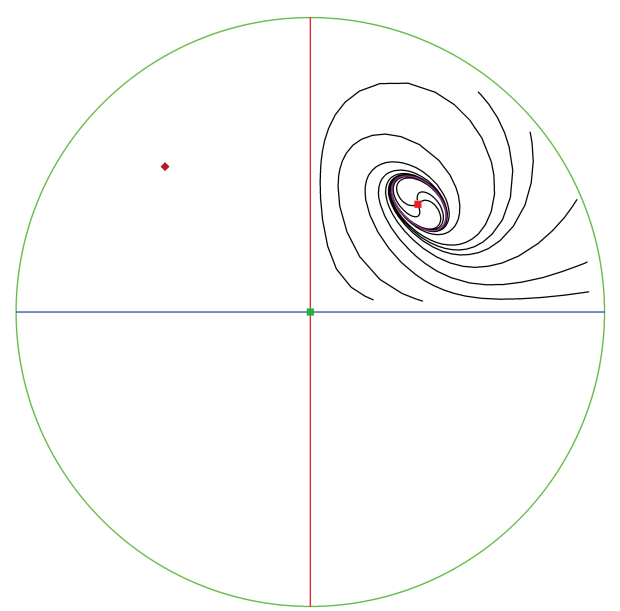

Fig. 2. The phase portrait in the Poincaré disc of the system (14), showing a limit cycle in the first quadrant

\section{Conclusion}

In this paper, a quintic Kolmogorov system with two parameters $\lambda$ and $\beta$ having $(1,1)$ as positive equilibrium point was investigated. By translation the coordinates of vector $(1,1)$ and rewritten the system in polar coordinates, we mainly shown that there is a sufficient condition for the existence of a limit cycle. Moreover, this limit cycle is non-algebraic in the case $\beta \neq 0$.

Finally, It is of interest to extend this study by answering to the following question: Is there a quartic or quintic Kolmogorov system that exhibit more than one non-algebraic limit cycle? This is left as a topic for future research.

\section{References}

[1] I.T.Al-Dosary Khalil, Non-algebraic limit cycles for parameterized planar polynomial systems, Int. J. of Math., 18(2007), no. 2, 179-189.

[2] A.Bendjeddou, A.Berbache, R.Cheurfa, A class of Kolmogorov system with exact algebraic limit cycle, Int. J. of Diff. Equa. Appli., 14(2015), no. 3, 159-165. 
[3] A.Bendjeddou, R.Cheurfa, On the exact limit cycle for some class of planar differential systems, Nonlinear Diff. Equ. Appl., 14(2007) 491-498.

[4] A.Bendjeddou, R.Cheurfa, Cubic and quartic planar differential system with exact algebraic limit cycles, Elect. J. of Diff. Equ., 2011(2011), no. 15, 1-12.

[5] A.Bendjeddou, J.Llibre, T.Salhi, Dynamics of the differential systems with homogenous nonlinearities and a star node, J. of Diff. Equ., 254(2013), 3530-3537.

[6] R.Benterki, J.Llibre, Polynomial differential systems with explicit non-algebraic limit cycles, Elect. J. of Diff. Equ., 2012(2012), no. 78, 1-6.

[7] S.Benyoucef, A.Bendjeddou, A class of Kolmogorov system with exact algebraic limit cycle, Int. J. of Pure and App. Math., 103(2015), no. 3, 439-451.

[8] S.Benyoucef, A.Bendjeddou, Kolmogorov system with explicit hyperbolic limit cycle, J. Sib. Fed. Univ. Math. Phys., 10(2017), no. 2, 216-222.

[9] R.Boukoucha, A. Bendjeddou, A Quintic polynomial diffential systems with explicit nonalgebraic limit cycle, Int. J. of Pure and App. Math., 103(2015), no. 2, 235-241.

[10] F.H.Busse, Transition to turbulence via the statistical limit cycle route, Synergetics, Springer-Verlag, Berlin, 1978.

[11] L.Cairó, J.Llibre, Phase portraits of cubic polynomial vector fields of Lotka-Volterra type having a rational first integral of degree 2, J. Phys. A: Math. and Theo., 40(2007), no. 24, 6329-6348.

[12] F.Dumortier, J.Llibre, J.Artés, Qualitative Theory of Planar Differential Systems, Universitex, Springer, Berlin, 2006.

[13] P.Gao, Hamiltonian structure and first integrals for the Lotka-Volterra systems, Phys. Lett. A., 273(2000), 85-96.

[14] A.Gasull, H.Giacomini, J.Torregrosa, Explicit non-algebraic limit cycles for polynomial systems, J. Comput. Appl. Math., 200(2007), 448-457.

[15] J.Giné, M.Grau, A note on : "Relaxation Oscillator with Exact Limit Cycles", J. of Math. Anal. and Appl., 324(2006), 739-745.

[16] J.Giné, M.Grau, Coexistence of algebraic and non-algebraic limit cycles, explicitly given, using Riccati equations. Nonlinearity, 19(2006) 1939-1950.

[17] J.Llibre, T.Salhi, On the dynamics of a class of Kolmogorov systems, App. Math. and Comput., 225(2013), 242-245.

[18] J.Llibre, J.Yu, X.Zhang, On the limit Cycle of the Polynomial Differential Systems with a Linear Node and Homogeneous Nonlinearities, Inter. of Bifu. and Chaos, 24(2014), no. 5, 1450065 .

[19] G.Lavel, R.Pellat, Plasma Physics, In Proceedings of Summer School of Theoreal Physics, Gordon and Breach, New York, 1975. 
[20] N.G.Llyod, J.M.Pearson, Limit cycles of a Cubic Kolmogorov System, Appl. Math. Lett., $\mathbf{9}(1996)$, no. 1, 15-18.

[21] N.G.Lloyd, J.M.Pearson, E.Sáez, I.Szántó, A Cubic Kolmogorov System with Six Limit Cycles, An Int. J. Comput. and Math. with Appl., 24(2002), 445-455.

[22] R.M.May, Stability and complexity in Model Ecosystems, Princeton, New Jersey, 1974.

[23] K.Odani, The limit cycle of the van der Pol equation is not algebraic, J. of Diff. Equ., 115(1995), 146-152.

[24] L.Perko, Differential equations and dynamical systems, Third edition, Texts in Applied Mathematics, 7, Springer-Verlag, New York, 2001.

[25] Xun-Cheng Huang, Limit cycles in a Kolmogorov-type Moel, Internat, J. Math.and Math Sci., 13(1990), no. 3, 555-566.

[26] Xun C.Huang, Lemin Zhu, Limit cycles in a general Kolmogorov model, Nonlinear Analysis, 60(2005), 1393-1414.

[27] Y.Yuana, H.Chenc, Ch.Du, Y.Yuan, The limit cycles of a general Kolmogorov system, $J$. Math. Anal. Appl., 392(2012), 225-237.

\title{
Класс квинтических колмогоровских систем с явным неалгебраическим предельным циклом
}

\author{
Ахмед Бенджедду \\ Кафедра математики, факультет наук \\ Университет Сетиф 1, 19000 \\ Алжир \\ Мохамед Гразем \\ Кафедра математики, факультет наук \\ Университет Бумердес, 35000 \\ Алжир
}

Различные физические, экологические, экономические и т.д. явления перекрываются планарными дифференциальными системами. Впоследствии некоторые исследования привлекут внимание $\kappa$ изучению предельных ииклов из-за их интереса к пониманию этих систем. Целью данной работь является исследование одного класса квинтических колмогоровских систем, а именно систем вида

$$
\begin{aligned}
& \dot{x}=x P_{4}(x, y), \\
& \dot{y}=y Q_{4}(x, y),
\end{aligned}
$$

где $P_{4}$ и $Q_{4}$ - квартичные полиномы. В этом классе наше внимание ограничено изучением предельного иикла в реалистическом квадранте $\left\{(x, y) \in \mathbb{R}^{2} ; x>0, y>0\right\}$. Согласно гипотезам доказано существование алгебрачческого или неалгебраического предельного иикла. Кроме того, этот предельный ицкл явно задан в полярных координатах. Некоторые примеры представлены для того, чтобы проиллюстрировать возможности применения нашего результата.

Ключевые слова: колмогоровские системы, первый интеграл, периодические орбиты, алгебраический и неалгебраический предельные иякль. 